C. Chiosi, E. Nasi ${ }^{+}$, G. Bertelli*

Istituto di Astronomia di Padova

+ Osservatorio Astronomico di Padova, and Istituto di Astronomia di Bologna

* Unità di Ricerca G.N.A./C.N.R. di Asiago-Padora

\title{
INTRODUCTION
}

Although it was known since a long time that very luminous blue and red stars may show evidence of mass outflow, it was the advent of the Copernicus satellite that clearly ascertained all $O B$ supergiants (and also the most luminous main sequence stars) are losing mass at rates that are significant for their evolutionary history. The observational information for blue luminous stars ( $O$, Of and WR) has been recently reviewed by Conti (1978), who discussed in some detail the data available for different spectral regions. The rates of mass loss inferred from these observations are estimated to be in the range $10^{-7}$ to $10^{-5} \mathrm{M}_{0} / \mathrm{yr}$ for $O B$ stars, and from $10^{-5}$ to $10^{-4} \mathrm{M}_{0} / \mathrm{yr}$ for WR stars. Several theoretical models have been proposed to explain those high mass-loss rates, and the high terminal velocities ranging from 1000 to $3000 \mathrm{~km} / \mathrm{sec}$. The two basic models are: the cool radiation pressure model, originally proposed by Lucy and Solomon (1970) and elaborated by Castor et al. (1975), in which the envelope is accelerated by momentum transfer from radiation to ions due to the ultraviolet line absorption; and the coronal model of Thomas (1973), and Hearn (1975), where the wind is sustained by gas pressure in a hot corona around the star. As both models do not completely account for the observations, several complementary modifications have been suggested. The nowaday situation is reviewed by Cassinelli and Lamers (1978), and Conti (1978).

On the side of red supergiants, both the observational and theoretical understanding of mass loss phenomenon unfortunately have not yet received the detailed systematic scrutiny afforded for luminous OB stars. The presence of circumstellar absorption lines in the spectra of cool stars is customarily thought of as indicator of mass outflow.

For lack of knowledge about the specific mechanism responsible of the mass loss, empirical mass-loss rates have

P. S. Conti and C. W. H. de Loore (eds.), Mass Loss and Evolution of O-Type Stars, 337-348.

Copyright $\odot 1979$ by the IAU. 
been estimated, Reimers (1975). However, the many uncertainties involved in the transformation of a circumstellar line profile into a mass-loss rate are such that the current esti mates for particular stars may differ by order of magnitude, Bernat (1977).

Amongst the others, one possible mechanism was suggested by Fusi-Pecci and Renzini (1975), who supposed the wind be originated by a hot corona produced by a non thermal process, such as the dissipation of the acoustic energy generated in the outer convective envelope.

The effect of mass loss, whatever might be the specific mechanism, on the evolutionary history of massive stars was argument of several independent theoretical investigations, Chiosi and Nasi (1974), Chiosi et al. (1978a,b), Czerny (1978), Dearborn et al. (1978), de Loore et al. (1977a,b). Most of these computations are limited to the central and shell H-burning, whereas only one attempt was made to follow the model evolution up and through the core He-burning. For lack of a completely satisfactory theory of mass loss in all evolutionary phases, semiempirical and parametrized approaches were adopted, and sequences of models whose mass simply decreases with time were computed. As long as the occurrence of mass loss is taken into account by such a hydrostatic treatment, the model computations are roughly equivalent even though different specific analytical relations for the massloss rate are adopted. Nevertheless, the sensitivity of the evolutionary results to the total amount of mass removed from the models during a given phase rises the problem of the con sistency of the mass-loss rate with the evolutionary phase under consideration. This is particularly true when semiempi rical mass-loss rates, inferred from the observational data, are used in computing the models. As an example of this, the adoption of mass loss rates for supergiant stars, presumably in a post main sequence stage, to compute losing mass models in central H-burning would certainly lead to overestimate the amount of mass lost during this phase.

In this note we will discuss the results of chiosi et al. $(1978 a, b)$, and perform a comparison with those of other authors. Moreover, by comparing the general features of the HR diagram of luminous $O B$ stars we try to put lower and upper limits to the mass-loss rate that has to be used in the model computations. The estimated mass-loss rate is confined within a range much narrower than the observational uncertainty. Finally we outline the current theoretical understan ding about the evolutionary status of the transition WR, Con ti (1976), and cast some light on the possibility that single stars might become single WR stars. 


\section{DESCRIPTION OF THE EVOLUTIONARY RESULTS}

Since quite exhaustive descriptions of the results about evolutionary models incorporating mass loss already exist in the literature, in the discussion below we will report only on the most salient points.

In the model calculations of chiosi et al. (1978a,b) the mass-loss rate was calculated from one of the three relations. At high effective temperature the evolutionary sequences were calculated using rates based on the theoretical study of Castor et al. (1975)

$$
\frac{d M}{d t}=\frac{L}{c v_{t h}} \frac{\alpha}{\Gamma}\left[\frac{1-\alpha}{1-\Gamma}\right]^{\frac{1-\alpha}{\alpha}}(K \Gamma)^{1 / \alpha}
$$

where the symbols have their usual meaning, $\alpha$ was taken as an adjustable parameter varying from 0.76 to 0.90 , Chiosi et al. (1978a). In the same range of effective temperature, Chiosi et al. ( $1978 \mathrm{~b}$ ) adopted a mass-loss rate based on the empirical formulation of Barlow and Cohen (1977)

$$
\frac{d M}{d t}=a\left(L / L_{0}\right)^{b}
$$

Two choices for a have been explored, namely $a=6.8 \quad 10^{-13}$ $M_{0} / y r$ and two times this value, $b$ was kept fixed and equal to 1.10. It is evident that the mass-loss rate of 0 stars of Barlow and Cohen (1977) was adopted. At low effective temperature, roughly below 3.8 in the logarithm, the mass-loss ra te adopted by Chiosi et al. (1978a) is based on the theoret $\bar{i}$ cal suggestion of Fusi-Pecci and Renzini (1975) of an acoustically driven wind. The rate is given by

$$
\frac{d M}{d t}=\varepsilon \frac{L_{a c}}{v_{\text {esc }} v_{s}}
$$

which is based on the momentum conservation. In the above re lation $L$ is the acoustic luminosity in solar units, vesc is a free parameter $\left(10^{-4}\right)$, Chiosi et al. (1978a). It must be said that the rates actually used in the computations were larger than those estimated by Reimers (1975) for less lu minous (massive) stars, although close to the recent evaluation of Bernat (1977). Semiconvection during core H-burning, shell H-burning, and He-burning was taken into account adopt ing the Schwarschild and Härm (1958) condition ( $\left.\nabla_{R}=\nabla_{0}\right)$. More details about the input physics and the computational procedure are in Chiosi et al. (1978a) and references quoted the- 
rein. Several sets of evolutionary sequences were computed for models of initial mass in the range $20 \mathrm{M}$ to $100 \mathrm{M}$ and with Pop I initial chemical composition ( $\mathrm{X}=0.700, \mathrm{Z}=0.82)$. All of them cover the core and shell H-burning phases, but with different laws for the mass-loss rate, either (I) or (2). while only three evolutionary sequences $\left(20,40,80 \mathrm{M}_{0}\right.$ of initial mass, and mass-loss rate given by eq. (l) with $\alpha=0.9$ ) were carried through the acoustic flux driven wind re gime for explorative purposes.

\subsection{The HR Diagram of the Core H-Burning Models}

Several novel features of the HR diagram of massive stars undergoing mass loss are evident by comparison with the evolutionary sequences at constant mass. With moderate massloss rates ( $10^{-7} \mathrm{M}_{0} / \mathrm{yr}$ for a $20 \mathrm{M}_{0}$ to $510^{-6} \mathrm{M}_{0} / \mathrm{yr}$ for a 100 $\mathrm{M}_{0} / \mathrm{yr}$ on the main sequence), the core $\mathrm{H}$-burning models are less luminous than they would have been without mass loss, and cover a wider range of effective temperature. With slight ly larger mass-loss rates the most massive stars, approxima.tely from $60 \mathrm{M}_{0}$ to $100 \mathrm{M}_{O}$, invert their path in the HR diagram, and long before the central H-exhaustion stage shrink towards the main sequence. This fact is mostly due to the lowering of the opacity in the outer layers when He-rich CNO processed material is brought to the surface by mass loss. This behaviour of the evolutionary sequences leads to quite a natural explanation of the observed upper boundary of the luminosity of $O B$ supergiants, decreasing with decreasing effective temperature, Hutchings (1976), snow and Morton (1976). It goes without saying that owing to the very short time sca le of the shell H-burning phase, the area of highest observa bility in the HR diagram is enclosed between the zero age main sequence and the locus of lowest effective temperature during the core H-burning phase. As those stars are very near to the main sequence, the location of the standard occupation area for core He-burning models in this range of mass ( 60 to $100 \mathrm{M}_{0}$ ) is too red to account them, stothers and Chin (1976). The discussion below will clarify that, even though in contrast with the conservative evolution, the core He-burning of losing mass models in this range of mass is found to take place near the main sequence, the rather short lifetime cannot account for the number of these stars which is comparable to what it would have had on the basis of a standard initial mass function. Moreover, the speculation is that core He-burn ing models of this initial mass should not appear as 0 , of but rather as WNT stars.

\subsection{Internal structure and semiconvection}

Several features of the internal structure of the core $\mathrm{H}$-burning models are worthy of mention because of their im- 
plications on subsequent evolution. They are: the mass exten sion of the semiconvective regions, the mass of the He core existing at the time of the core He-ignition, the profile of chemical composition throughout the models.

As it was firstly noticed by Chiosi and Nasi (1974), and later discussed to some extent by Chiosi et al. (1978a,b), no ne of the evolutionary sequences computed taking into account mass loss shows the presence of semiconvection during the entire core H-burning phase: Semiconvection and full intermedia te convection are restored later, although to a much less extension than it would have occurred in constant mass models. The analysis of Chiosi et al. (1978b) also clarifies that mass-loss rates as low as those given by Barlow and Cohen (1977) for 0 stars, likely constitute an upper limit for the existence of semiconvection in massive stars. With the occur rence of mass loss, the mass of the He core is significantly smaller than for constant mass models, and decreases with in creasing mass-loss rate. This result is relevant for the eva luation of the yield of heavy elements per stellar generation and correlated galactic chemical evolution, Arnett (1978). Adopting the new $M_{g}\left(M_{j}\right)$ relation for losing mass models, Chio si and Caimmi (1978) and Chiosi (1978a) estimated a significantly lower yield of heavy elements. It must be also emphasized that this result depends only on the occurrence of mass loss during the main sequence phase, and is not fraught with the many uncertainties inherent to mass loss during the red phases.

\subsection{The Problem of the Mass Determination}

In presence of mass loss the evolutionary tracks do not coincide with lines of constant mass in the HR diagram, and the current procedure of assigning the mass to a single star by means of either a mass-luminosity relation or the use of evolutionary tracks is no longer valid. This problem was discussed to some extent by Chiosi et al. (1978a). No attempt however was made to re-estimate the mass of individual stars in the list of Snow and Morton (1976). The possibility of a substantial difference between the actual mass of a star in presence of mass loss and that it would have been assigned by a standard procedure was taken into account by Abbott (1978) discussing the relationship between the terminal and the escape velocity. Amongst the others, the adoption of more realistic masses for stars undergoing mass loss revealed that terminal velocities can be fitted by the empirical rela tion $v_{\infty} \approx 3 v_{e s c}$, in agreement with the predictions from the theory of radiatively driven stellar winds of Castor et al. (1975). Finally, Chiosi et al. (1978a) showed how the uncertainty in the mass determination is not very severe for stars close to the main sequence and of rather low mass, approxima tely below initial $45 \mathrm{M}_{0}$, but can be as large as $40-50 \%$ in 
the case of the most luminous stars.

1.4. The Isochrones

The determination of the age of star clusters by means of the isochrones in the HR diagram involves the bolometric magnitude $M_{b}$ and/or the spectral type $S p$ of the turn off point of the core H-burning sequences, Barbaro and Chiosi (1973). These two parameters are known to correlate primarily with the age, and secondly to the initial chemical composition $X$ and $Z$. The effect of mass loss is to lower the ages for the same $M_{b}$ and $S p$ and fixed chemical composition below those allowed by constant mass evolution. This fact could partially remove the well known discrepancy between the kine matic expansion ages and nuclear ages (estimated from evolutionary tracks).

\subsection{The Generator Function of the Mass-Loss Rate}

The comparison of the present results with those of other authors, Czerny (1978), and de Loore (1977a,b) was performed by Chiosi et al. (1978b). To this purpose several basic rela tionships among fundamental variables $\left[\mathrm{LOg}\left\langle\mathrm{L}_{\mathrm{O}} / \mathrm{L}_{O}\right\rangle \sim \operatorname{LOg}\langle\dot{M}\rangle\right.$, $\operatorname{LOg}\left(M_{f} / M_{O}\right) \sim \operatorname{LOg}\langle\dot{M}\rangle$, and $\left.\operatorname{LOg} t_{H} \sim \operatorname{LOg}\langle\dot{M}\rangle\right]$ were constructed, aimed to provide a theoretical network to which compare the observations. Owing to this, the area covered in the HR diagram by losing mass models in core H-burning, the amount of mass lost by the models during the core H-burning phase, the variation of the core H-burning lifetime, and the surface chemical abundance have been compared as functions of the initial mass, mass-loss rate, and mass-loss rate relation. This analysis allowed us to put plausible constraints on the mass-loss rate and its dependence upon fundamental stellar parameters to reproduce the observed distribution of losing mass OB stars in the HR diagram. A good agreement is achieved by models having mass-loss rates intermediate to those of the set $\alpha=0.83$ and $\alpha=0.90$ of Chiosi et al. (1978a). The comparison of the theoretical rates to those inferred from the observations, Barlow and Cohen (1977), is made introducing the concept of the generator function of the mass-loss rate dependence. In fact, it goes without saying that rate and luminosity of losing mass stars are conditioned by the occurrence of mass $108 s$ in their previous history. Hence the lumi nosity to mass-10ss rate dependence, such as suggested by the observations, might be somewhat different from the one actual ly involved in the mass loss process, and responsible of the observed features. In virtue of this, and taking into account that the maximum probability of observing a losing mass star is expected at some intermediate stage of central H-burning, Chiosi et al. ( $1978 \mathrm{~b}$ ) correlated the experimental rates of Barlow and Cohen (1977) to theoretical values intermediate 
between those of the sets $\alpha=0.83$ and $\alpha=0.90$ of Chiosi et al. (1978a) when the mass-loss rate of $\zeta$ Pup is taken as a basic constraint.

\subsection{The Core He-Burning Phase}

Three evolutionary sequences of the set $\alpha=0.90$, namely with initial masses $20,40,80$ Mo were continued through the red stages in the HR diagram, taking into account mass $10 s$ by acoustic flux mechanism of Fusi-Pecci and Renzini (1975), and carried up to advanced stages of central He-burning, which takes place at high effective temperature, Chiosi et al. (1978a). Although the mass-loss rates of the acoustic flux driven wind phase were higher than those extrapolated from the empirical relation of Reimers (1975) for lower lumi nosities, still the results below can be used as exploratory indication of the effect of mass loss on core He-burning models.

Current ideas about the problems of the core He-burning phase of conservative evolution have been recently reviewed by Chiosi (1978b). The location in the HR diagram of conservative models in stationary central He-burning is known to depend primarily upon the intermediate convective instability through its effect on the chemical profile of the models, and secondly on details of the input physics. In the case of the Schwarzschild and Härm (1958) condition, the results can be schematically described as follows. In the range of mass 20 to $40 \mathrm{M}_{0}$ the models ignite the central He-burning as blue supergiants, slowly moving redwards in the HR diagram. Two areas of stationary burning with nuclear time scale, can be identified, blue and red separated by a yellow region of secular instability, thermal time scale. The relative lifetime spent in the blue and red stages is not unique with the stel lar mass, but is known to depend also on several factors of the input physics, Chiosi (1978b).

Models whose mass is greater than roughly $40 \mathrm{M}_{0}$ do not show the blue phase, but after a rapid run toward low effective temperature regions spend the whole core He-burning as red supergiants. This picture was understood mostly in terms of the effect of the H-profile on the model location in the HR diagram, Chiosi $(1978 \mathrm{~b})$ and references quoted therein. The occurrence of mass loss during core $H$ and He-burning phases somewhat affects the above schematization, and the models of Chiosi et al. ( $1978 \mathrm{a}, \mathrm{b})$ allow to draw several preliminary re marks.

Models with significant loss of mass during the core Hburning phase, no matter of their initial mass, move redward on a very short time scale. Further mass loss at high rate $\left(\sim 10^{-4} \mathrm{M}_{\odot} / \mathrm{yr}\right)$, via the specific mechanism of acoustic flux driven wind in our computation, in the region of low effecti ve temperature forces the models back into the blue supergiant 
area. The bluewards movement seems to take place once the fractionary mass of the He core, $M_{H e} / M$, gets larger than 0.6 , being the models still far from the thermal equilibrium condition. Hence the stationary He-burning in the core can initiate only after the models have reappered as blue supergiants. Later on they slowly move to higher and higher effec tive temperature as the central He-content is burnt out. The fate of models with smaller mass-loss rate at low effective temperatures, being not yet computed is still unknown, although we might expect a qualitatively similar behaviour.

Moderate mass loss during the phase of central H-burning gives quite a different picture. Models with initial mass greater than $40 \mathrm{M}_{O}$ do not start the stationary central Heburning at high effective temperature, but on a short time scale move redward. Their subsequent evolution in presence of mass loss resembles to that of the previous case. On the con trary models with initial mass $\leqslant 40$ Mo have the stationary co re He-burning as blue supergiants, while slowly moving redward. The phase of low effective temperature mass loss should be reached for these models in a very advanced stage of central He-burning. Their later evolution not yet computed is still uncertain.

We would like to outline as the most salient result of these computation the discriminatory effect of mass loss on the movement direction in the HR diagram for core He-burning models. Moderate mass loss (blue and red stages) causes the models in stationary central He-burning to move from blue to red. On the contrary high mass loss (blue and red stages) ma kes the models in stationary He-burning to move bluewards. The preliminary nature of these computations makes the core He-burning phase of losing mass models still fraught with ma ny uncertainties, and demands a deeper investigation.

\subsection{The Blue, Yellow and Red Supergiants}

The comparison of the predicted zone of occupation for stars evolving at constant mass and in core He-burning phase with the experimental distribution of blue, yellow and red su pergiants in the HR diagram was widely discussed by stothers and Chin (1976), and references quoted therein. It was clear from that analysis that in the case of the schwarzschild and Härm criterium the models were too cool compared with the bulk of blue stars, and also they predicted too many red supergiants of mass greater than $30 \mathrm{M}_{\vartheta}$. On the contrary, one of the most remarkable features of the HR diagram of supergiant stars in the galaxy is the lack of the very luminous red supergiants $\left(M_{b}<-8\right)$. Stothers and Chin (1976) suggested that either different opacities or inclusion of mass loss in model computations could remove the above difficulties. Models of Chiosi et al. (1978a) showed that the core He-burning phase of losing mass models firstly covers a broader area of the HR 
diagram, greatly improving the agreement with the observation, secondly that stars more massive than $50 \mathrm{M}_{0}$ on the main sequen ce, which suffer substantial mass loss during the core H-burn= ing and later phases, do not reach the Hayashi line, thus never becoming red supergiants. It is worth noticing that this result is due to the effect of mass loss mostly during core H-burning rather than later phases. Alhtough preliminary, the se results lend some support to the idea that mass loss from massive stars could actually be a clue to achieve a satisfac tory understanding of the distribution of blue, yellow and red supergiant stars in the HR diagram.

\section{THE WR STARS}

The majority of WR stars are known members of binary sy stems, but whether or not they all are binaries is not yet firmly established. The current theoretical understanding of confirmed binary WR stars recognizes in the mass exchange pro cess the mechanism responsible for producing a WR object although many questions are still left unsolved. However, as a number of well studied objects (e.g. HD 192163, HD 50896) has no indication of binary nature, we must consider the possibi lity that some of the WR stars are single objects and seek other processes than mass exchange. Conti (1976) made the sug gestion that 0 stars would become $0 f^{\prime} s$ if substantial wind we re to exist. With increasing strength of the mass outflow, Of's would transform into "transition WR stars". Later, if further mass loss were still to occur, as suggested by the ob servations, Conti (1978) and references quoted therein, the transition WR's would evolve into classical WR stars. It is evident that a temporal evolutionary sequence is suggested to exist among 0 , Of and all WR types. The discussion below will propose a slightly different picture which is perhaps able to remove part of the difficulties present in Conti's (1976) sug gestion. Reviews of the general properties of WR stars are by Conti (1976) and Paczynski (1973). However a few basic points are worth mentioning here, as they are relevant to our discus sion. The position of WR stars in the HR diagram was discussed by Conti (1976). The majority of WR's are located in the box $-8>M_{b}>-9,4.4<\operatorname{LOg} \mathrm{T}_{e}<4.7$, (the bolometric corrections and the color index-effective temperature scale may however intro duce a considerable uncertainty), on the contrary, the transi tion WR's otherwise classified as WN7/WN8 are clearly apart from other subclasses as they are situated at higher luminosities and have average hotter effective temperatures. As far as the chemical composition of the surface is concerned, there is little evidence of hydrogen in most WR's, the $N(H) / N(H e)$ ratios being in the range 1.-2. for WN7/WN8 types, roughly 0.4 for WN4, WN6 and very close to zero for WN5. It appears also that WN stars have more $N$, whereas WC stars have more C 
and 0 at the surface. Elementary considerations suggest that layers coming from regions of internal nucleosynthesis (CNO for WN stars, and $3 \alpha$ for WC stars) are to be exposed at the surface to explain those chemical compositions. Paczynski (1973) suggested the peeled onion skin model that relies on the existence of mass loss. Very presumably WC's should be more evolved than WN's. The statistics about the frequency of WR's among OB stars was elaborated by Smith (1973), and by Moffat and Seggewiss (1978) for the transition WR's in parti cular. Chiosi et al. (1978a) in the light of their results on the evolution of massive stars with mass-loss in blue and red phases rediscussed the problem of the theoretical interpreta tion of WR stars.

Before proceeding further it must be emphasized that Chiosi's et al. (1978a) scenario was aimed to suggest a plau sible explanation of single WR's, and is not in conflict with the mass exchange mechanism in binary systems. In their sceng rio, all single WR stars are in the core He-burning phase, whereas the existence of different classes is interpreted in terms of the progenitor mass and overall effect of mass loss. As far as the progenitor star is concerned, single stars with initial mass smaller than 20-25 M० would never undergo a WR phase as a consequence of mass loss by stellar wind in the course of their evolution. The computations in fact indi cate that very little mass is lost on the whole, preventing the stars from showing very low $H$ content at the surface. They should behave as standard main sequence and supergiant stars but with minor changes caused by the existence of mass loss (e.g. anomalies in the surface chemical composition, Luck $1977 \mathrm{a}, \mathrm{b}$; 1978 ).

Single stars with initial mass in the range 20-25 Mo to 40-50 $M_{\theta}$ would first appear as WN and later as WC during the most advanced stages of central He-burning. This is due to the combined effect of mass loss during the core H-burning (radiation pressure) and He-burning (acoustic flux driven wind, and radiation pressure). When the stationary He-burning in the core is initiated, the models run blueward in the HR diagram, still having a rather thin H-rich envelope at the top of the He core. This envelope can be easily lost by the star on a time scale shorter than the He-burning lifetime if mass 1088, presumably by radiation pressure, were to occur at a rate compatible with the current estimate for WR stars $\left(10^{-5}\right.$ to $\left.10^{-4} \mathrm{M}_{0} / \mathrm{yr}\right)$. It was also suggested that single WC stars would correspond to the fraction of the core He-burning lifetime after the removal of the whole H-rich envelope and before the central exhaustion stage. WN stars would occur of course before the H-rich envelope is lost and after the models have already moved to high effective temperature ( Log $_{\mathrm{e}} \mathrm{T}_{4} .3$ ). In fact, although the models were not carried until the central He-exhaustion stage, still they went very near to the area of classical WN and WC stars. Finally, stars of initial 
mass greater than 40-50 Mo would also undergo a WR phase during their core H-burning phase but appearing only as transi tion WR's, as they would die before the H-rich envelope is completely lost. The ultimate motivation of this picture rests on the high efficiency of mass loss during core H-burn ing phase in massive stars. The numerical computations of Chiosi et al. ( 1978 a) seem in fact to indicate that when the mass of the H-rich envelope reduces below $40 \%$ of the total mass no red models are possible; mass loss at low effective temperature loses part of its importance. A relative signifi cant H-rich envelope is left and the time scale necessary to remove it is longer than the core He lifetime. Later phases are unrelevant to this purpose owing to their extremely short lifetime in presence of the neutrino cooling. Our predictions for the transition WR's is somewhat supported by the observa tional analysis of Moffat and Seggewiss (1978). In conclusion, the transition WR's have evolved from progenitors of higher mass than all other WR stars and moreover in view of their distinct luminosity gap in the HR diagram very presumably they would not evolve into other subclasses.

A further speculation suggested by the above picture is the ultimate fate of stars in the range of mass 20-25 Mo to 40-50 $\mathrm{M}_{0}$. If we ask the question of the optical appearance of a SN produced by the explosion of stars deprived of their H-rich envelope, their spectrum could resemble to SNI spectrum. At the time being there is no strong observational evi dence of this category of type I supernovae, and further investigation is necessary to elucidate the point.

\section{REFERENCES}

Abbott, D.C.: 1978, preprint

Arnett, D.W.: 1978, Astrophys. J. 219, 1008

Barbaro, G., Chiosi, C.: 1973, Astron. Astrophys. 28,7

Barlow, M.J., Cohen, M.: 1977, Astrophys. J. 213, 737

Bernat, A.P.: 1977, Astrophys. J. 213, 756

Cassinelli, J.P., Lamers, N.J.G.L.M.: 1978, preprint

Castor, J.I., Abbott, D.C., Klein, R.I.: 1975, Astrophys. J. 195, 157

Chiosi, C.: 1978 a, preprint

Chiosi, C.: 1978b, in Proc. I.A.U. Symposium $\mathrm{N}^{\circ} 80$, Washington D.C., in press

Chiosi, C., Caimmi, R.: 1978, preprint

Chiosi, C., Nasi, E.: 1974, Astron. Astrophys. 34, 355

Chiosi, C., Nasi, E., Sreenivasan, S.R.: 1978a, Astron. Astrophys. 63, 103

Chiosi, C., Nasi, E., Bertelli, G.: 1978b, submitted to Astron. Astrophys.

Czerny, M.: 1978, preprint

Conti, P.S.: 1976, Mem. Soc. Roy. Sci. Liège $6^{\circ}$ Serie 9, 193 
Conti, P.S.: 1978, Ann. Rev. Astron. Astrophys., in press

Dearborn, D.S.P., Blake, J.B., Haineback, K.L., Schramm, D.N.: 1978, Astrophys. J. in press

de Loore, C., De Grève, J.P., Lamers, H.J.G.L.M.: 1977a, Astron. Astrophys. 6i, 251

de Loore, C., De Grève, J.P., Vanbeveren, D.: 1977b, preprint Fusi-Pecci, F. Renzini, A.: 1975, Mem. Soc. Roy. Sci. Liège $6^{\circ}$ Serie 8,383

Hearn, A.G.: 1975, Astron. Astrophys. 40, 355

Hutchings, J.B.: 1976, Astrophys. J. 203, 438

Luck, R.E.: 1977a, Astrophys. J. 212, 743

Luck, R.E.: $1977 \mathrm{~b}$, Astrophys. J. $\frac{218}{19}, 752$

Luck, R.E.: 1978, Astrophys. J. $2 \overline{19}$. 148

Lucy, L.B., Solomon, P.M.: 1970, Astrophys. J. 159, 879

Moffat, A.F.J., Seggewiss, W.: 1978, preprint

Paczynski, B.: 1973, in Wolf Rayet and High Temperature Stars, Ed. M.K.V. Bappu and J. Sahade (Dordrecht, Reidel)

p. 143

Reimers, D.: 1975, Mem. Soc. Roy. Sci. Liège $6^{\circ}$ Serie 8,366

Schwarzschild, M., Härm, R.: 1958, Astrophys. J. 128, 348

Smith, L.F.: 1973, in Wolf Rayet and High Temperature Stars,

Ed. M.K.V. Bappu and J. Sahade (Dordrecht, Reidel)

p. 15

Snow, T.P., Morton, D.C.: 1976, Astrophys. J. Suppl. 32, 429

Stothers, R., Chin, C.W.: 1976, Astrophys. J. 204, $47 \frac{32}{2}$

Thomas, R.N.: 1973, Astron. Astrophys. 29, 297

\section{DISCUSSION FOLLOWING CHIOSI, NASI and BERTELLI}

de Loore: How can you explain that during the acoustic flux regime mass loss the luminosity increases? I should presume that due to entropy losses the luminosity drops. I would refer to our computations for a 30 Mo star, lowered to 17.8 Mo owing to stellar wind, where at the red giant stage a mass loss rate of $4.10^{-3} \mathrm{Moyr}^{-1}$ was applied. There the track goes down as it should. I think that energy losses should be taken into account.

Chiosi: I agree with you and I believe that a fully hydro-dynamical approach would be much more appropriate.

Mazurek: Type I supernovae have large expansion velocities, implying a relatively low mass ( $\left.1 M_{0}\right)$. You are making Type I supernovae out of massive ( 50 Mo) stars. How can you justify this?

Chiosi: In my picture I was trying to suggest that a new kind of Type I supernovae might be originated by rather massive stars say between 30 to 60 Mo. This suggestion is however very speculative as we do not yet have any observational support for this. The statistics should be in any case rather poor 